\title{
Examining Patient Perspectives on Weight Management Support in the Primary Care Setting
}

\author{
Kate Bloom, et al. [full author details at the end of the article]
}

Published online: 14 July 2018

\begin{abstract}
Obesity affects more than one-third of Americans and is a leading cause of preventable death. Integrating patient perspectives into obesity treatment can help primary care providers (PCPs) intervene more effectively. In this study, we describe patients' experiences with PCPs concerning the diagnosis and treatment of obesity and offer suggestions for patient-centered care in weight management. We conducted four focus groups with patients of a university medical system-associated family practice who had a BMI $\geq 30$. Interview questions addressed general weight management perceptions and preferences for weight management support in a primary care setting. Patients completed a brief demographic survey at the conclusion of the group. Four authors independently coded focus group notes to identify themes and determine saturation using qualitative thematic analysis. We resolved discrepancies by team discussion. Thirty primary care patients participated, of whom 23 were female and whose average age was 50 . Twenty-four had attempted to lose weight in the past 12 months and had discussed management with their providers. Analyses identified four themes regarding weight management in a primary care setting: motivation and weight management, the provider-patient relationship, desire for concrete weight loss plans, and limitations of the primary care setting. Motivation was named as a weight management obstacle. Participants felt that PCPs need to be partners in weight management efforts and also recognized limitations of PCP time and expertise. They endorsed an integrated behavioral approach that includes physical activity and nutrition support. Improving PCP delivery of evidence-based treatment for obesity will lead to increased patient attempts to lose weight. Incorporating patients' desires for concrete plans, ongoing support, and referral to integrated service (e.g., nutritionists, care managers, behavioral health providers) programs can increase patient engagement and success. The chronic disease care and Patient Centered Medical Home models offer guidance for ensuring sustainability of weight management services.
\end{abstract}

Keywords Weight management - Obesity · Weight reduction · Primary care Clinician-patient communication and relationship - Qualitative methods $\cdot$ Focus groups 


\begin{tabular}{ll}
\multicolumn{2}{l}{ Abbreviations } \\
BMI & Body mass index \\
PCP & Primary care provider \\
PCMH & Patient Centered Medical Home \\
UNC FMC & University of North Carolina Family Medicine Center \\
USPSTF & United States Preventive Services Task Force \\
US & United States
\end{tabular}

\section{Introduction}

More than one-third of adults in the United States (US), or over 70 million people, meet the clinical definition of obesity (i.e., a body mass index $\geq 30$; Centers for Disease Control and Prevention, 2010). As the prevalence of obesity has risen steadily over the last few decades (Centers for Disease Control and Prevention, 2014), providing adequate weight management support in the primary care setting is of increasing importance (Centers for Disease Control and Prevention, 2010).

The US Preventive Services Task Force (USPSTF) recommends that all providers screen adults for obesity and refer those who are obese to "intensive, multicomponent behavioral interventions" (LeBlanc, O'Connor, Whitlock, Patnode, \& Kapka, 2011a, b; USPSTF, 2012). Recommended effective interventions include 12-26 comprehensive sessions per year with a physician, nutritionist, or other provider (USPSTF, 2012). Participants in such interventions lost an average of $6 \%$ of their baseline weight relative to control groups (USPSTF, 2012). Unfortunately, the USPSTF was not able to determine which specific components of programs were most effective (e.g., group sessions, individual sessions, addressing barriers to change; USPSTF, 2012). However, a survey of over 600 patients who were overweight or obese showed that those whose primary care provider (PCP) discussed weight loss with them were significantly more likely to attempt to lose weight and to achieve clinically significant weight loss (Gudzune, Bennett, Cooper, \& Bleich, 2014), showing the important role played by PCPs in patients' weight loss efforts.

Despite this finding, research shows PCPs infrequently address obesity in practice (Campos, 2014; Davis, Emerenini, \& Wylie-Rosett, 2006; Hebl \& Xu, 2001; Wadden et al., 2000). Because weight loss counseling for patients is not a nationwide standard, providers may miss counseling opportunities (Davis et al., 2006). In a study of 456 patients diagnosed with obesity and their physicians, physicians reported weight-related discussions happening more frequently than did patients (Greiner et al., 2008). Another study of 122 physicians found that only $42 \%$ of physicians seeing patients with obesity and only $35 \%$ seeing overweight patients (BMI = 25-29) discussed weight loss (Hebl \& Xu, 2001). Physician barriers to weight loss counseling include time constraints, insurance and reimbursement uncertainty, negative stereotypes surrounding patients with obesity, and beliefs that patients may not want to discuss their weight (Davis et al., 2006). 
When patients with obesity seek weight management counseling from their PCP, they report varying levels of satisfaction with these interactions. They frequently report provider bias, which encompasses experiences such as feeling a lack of respect or that they are not taken seriously (Puhl \& Heuer, 2010). This may lead patients to delay seeking health care or to cite their weight as a barrier to getting appropriate care (Puhl \& Heuer, 2010). In addition, those who perceived negative judgment from their provider were significantly less likely to achieve clinically significant weight loss relative to those who did not perceive that they were judged negatively by their provider (Gudzune et al., 2014).

In sum, PCPs do not provide consistent weight management care. Moreover, it is unclear what weight management services patients actually want and how they would like to structure interactions with their PCP. This information would allow providers to integrate patient perspectives and experiences into obesity treatment programs to enhance program effectiveness.

This qualitative study, conducted at a university health system-related family medicine clinic, examined patient perspectives on obesity. We sought to understand: (1) views of patients with a diagnosis of obesity on weight management, (2) patients' experiences addressing weight management with their providers, and (3) ways PCPs can create a supportive health care environment while caring for patients with weight management issues.

\section{Methods}

\section{Study Area}

The UNC Family Medicine Center serves approximately 18,000 patients from diverse backgrounds and is a Patient Centered Medical Home with integrated care for behavioral health, including a psychologist, registered dieticians, and tobacco use treatment specialists. Approximately 60 PCPs include attending and resident physicians as well as several nurse practitioners. Providers may discuss weight management strategies with patients during appointments and make referrals to an in-house nutritionist or outside bariatric surgery program, but there is no comprehensive program to address weight.

\section{Study Approach}

We conducted focus groups to collect qualitative data addressing our research questions. This approach allowed us to elicit discussions around the common experience of obesity. Qualitative research study samples are not comparable to quantitative samples and allow researchers to collect and extract data that contains deeper and more meaningful responses from a fewer number of participants (Miles \& Huberman, 1994). We asked questions about participants' perspectives on, and experiences with, weight management in primary care, a less sensitive topic than weight itself, making focus groups an effective format. 


\section{Sampling and Recruitment}

The clinic administration generated a list of all patients 18 years or over with a BMI of $\geq 30$. We stratified this list by smoking status, gender, and race to increase likelihood of participation by the patient population. We held separate groups by smoking status, thinking that similar behavioral interventions are related to both conditions (i.e., obesity and tobacco use) and may influence one another; those quitting tobacco often fear weight gain (Vallis, Piccinini-Vallis, Sharma, \& Freedhoff, 2013). Participants visited the clinic at least once in the previous 12 months and were fluent in English. We randomly selected individuals within the strata to receive a focus group invitation. Patients were recruited via email when there was an email address available and postal mailing if no email address was provided or if the email returned undeliverable. We recruited sufficient patients to have an average of seven patients per group.

Potential participants completed a brief online Qualtrics eligibility and availability screening questionnaire or left contact information on a secure voicemail line if they preferred a telephone screening.

\section{Focus Groups}

We conducted four focus groups from December 2014 to January 2015. To ensure a neutral setting, we conducted the groups in a wellness center affiliated with the university but not directly within the family medicine clinic. Each focus group lasted 90 min. Two authors with qualitative research experience and masters-level qualitative methods training co-moderated each focus group and two other authors (with the same experience and training) served as note-takers and audio recorded the groups. We developed a semi-structured interview guide, which asked about general perceptions of weight management, past experiences with primary care providers concerning weight management, and what participants would want from a weight management program in a primary care setting. These questions were developed from a review of the literature and focused on information we wanted to learn to inform a planned pilot weight management program. We held a pilot focus group with family medicine department staff and incorporated suggestions into the final guide.

At the conclusion of each focus group, participants filled out a brief paper survey that asked basic demographics, e.g., age, race/ethnicity, sex; several behavioral questions, e.g., smoking status, weight loss attempts in past 12 months; and whether or not a PCP had initiated a conversation about weight (Table 1). The purpose of this survey was to be able to describe group participants. Each participant received a $\$ 30$ gift card for participation. The Institutional Review Board for human subjects at the University of North Carolina at Chapel Hill approved the study.

\section{Data Analysis}

Following each focus group, the interview team met to discuss overall impressions, non-verbal participant behaviors, and major themes that emerged. 
Table 1 Focus groups participant characteristics $(\mathrm{n}=30)$

\begin{tabular}{lc}
\hline & $N(\%)$ \\
\hline Mean age, years $(S D)$ & $50.3(11.5 \%)$ \\
Female & $23(76.7 \%)$ \\
Non-smoking & $21(70 \%)$ \\
Race & $13(43.3 \%)$ \\
White & $12(40 \%)$ \\
Black & $3(9.9 \%)$ \\
Other & $1(3.3 \%)$ \\
More than one race & $24(82.8 \%)$ \\
Tried to lose weight in past 12 months & $23(82.1 \%)$ \\
PCP-initiated conversation about weight management & \\
\hline
\end{tabular}

We reviewed the focus group audio recordings and added verbatim quotes to our original extensive focus group notes. Each of the four interviewers reviewed all focus group notes independently and identified themes. We met again to reach consensus on themes and develop a codebook. Four authors independently coded the focus group notes using these codes, resolving discrepancies in coding by team discussion. As we coded the focus group notes, we determined that we had reached thematic saturation by the end of the fourth focus group (i.e., no new themes emerged; Rice \& Ezzy, 1999). We used Dedoose Qualitative Data Analysis software to develop the codebook, manage the coding process, and assist with data analysis.

\section{Results}

\section{Participants}

A total of 30 primary care patients attended the four focus groups (range 4-11 people in each group). The majority was female (23), with a mean age of 50 $(S D=11.3)$. Forty three percent were White and 40 percent African American. Most participants (24) had tried to lose weight in the past 12 months and had engaged in a PCP-initiated conversation about weight management (23). Seventy percent did not smoke.

We found four themes in the focus groups: motivation and behaviors for longterm weight management, the patient-provider relationship and weight management, need for concrete weight-loss guidance, and limits of the primary care setting.

\section{Theme 1: Motivation and Behaviors for Long-Term Weight Management}

Overwhelmingly, participants expressed understanding of weight management principles, but other factors kept them from being motivated to make changes. 
Intrinsic factors participants mentioned included needing initial weight loss success for motivation to continue, enjoyment of "good" food, and dislike of exercise. One participant referred to intrinsic motivation for weight management as a "blessing" and a "gift," suggesting it was something only some people possessed.

Extrinsic factors mentioned related to accountability to a partner or a group to help encourage new behavior: "You want to do it, but it is hard to motivate yourself. If you have someone that's helping you, it's so much easier." "There is a group piece of Weight Watchers that you could bring into a weight management clinic that would be helpful." Many participants wanted assistance fitting weight management behaviors (both exercise and diet) into their current life without feeling overwhelmed: "With so many other things going on, the last thing you need is an eating schedule. I can say what I think weight management looks like, but I don't know how to live it."

\section{Theme 2: Patient-Provider Relationships and Weight Management}

Participants spoke about positive relationships with their PCPs, even in relation to weight management. They wanted their PCP to be involved in their weight management efforts, with many stating the importance of "medical supervision." Participants wanted consistent positive reinforcement and follow-up from their PCP: "It feels good when someone tells you you're doing good." Interest in PCP involvement related to the motivation and accountability reported in Theme 1. Participants thought their PCP's knowledge of their weight management efforts would bring additional accountability and wanted to be asked about their efforts at follow-up visits:

Why can't they say, 'Okay, well we talked about you needing to start walking. How much have you walked in the last month?'”

"If I had lost some weight, the first thing I expected [my PCP] to say when he walked in was 'Hey, you lost weight.' I had to say to [my PCP], 'Look, I lost some weight!

Communication with their PCP between office visits was important to participants. Some felt it helpful to be able to call or email their PCP. Others felt they would benefit from following up with brief questions about the weight management issues they discussed with their PCP: "When you leave the office, the responsibility is all yours...There is no accepted person to follow up with or to call." Furthermore, participants wanted to feel as if their PCP was a partner in their weight management efforts: "We have to work together and it's a partnership. You're helping me to help myself, so it's a collaboration."

Most participants felt the time limits of their primary care appointments constrained weight management discussions and PCP-relationship development, although they understood why time limits were in place:

I like my doctor, but I feel like when we talk about weight management, it's always very hurried and rushed and then she has to go on to see someone else. I never feel like she has time to give thought to my particular issue. 
Another participant likened her primary care appointments to "speed dating," saying, "They're going from one room to the next... and it's like they're not even hearing you...It's hard sometimes to talk about your health because your health is so personal." These limitations meant weight was only broached when it caused other health issues or if the participant initiated the conversation. Participants thought weight management issues deserved more time.

Finally, participants needed their PCPs to see them as a whole person. For some participants, this meant recognition of them as more than a constellation of symptoms: "She was looking at me not as a person, but as a statistic." Others felt their PCPs were too focused on weight loss and not focused on other issues that might be contributing to their weight: "Even after I mentioned to her about certain stressors that were taking place in my life, she never even touched on that... She was just like a recorder, 'If you lose weight and exercise...",

Other participants had issues when weight gain was a medication side effect. They wanted upfront information about weight gain as a potential side effect, but reported that their PCP was often not aware of it. One participant reported her PCP was "shocked" when the medications caused weight gain.

Most participants had positive experiences bringing weight management suggestions to their PCP. These participants felt they were being taken seriously: "I could say to [my PCP] I'm doing investigations on this thing and even if he probably thinks it's silly he doesn't say that. He makes me feel like I'm important and should keep doing it." Participants were encouraged by these interactions and felt this was a valuable part of their patient-provider relationship.

\section{Theme 3: Need for Concrete Weight Loss Guidance}

Central to the participants' relationship with their PCP was how capable they thought their PCP was in providing weight management assistance. What participants wanted from their PCPs was often in contrast to what they received.

Participants desired clear weight management guidance from their PCP. They wanted to discuss specifics and to develop tailored, usable diet and exercise plans. As mentioned above, many participants understood weight management but had trouble making behavior changes. They also wanted their PCP to provide estimations of the results they could expect from such a plan:

It would be very helpful for me if someone were to present to me, 'Here is a diet plan. This is what you need to eat Monday, Tuesday, Wednesday, Thursday, Friday, and if you do this, you're going to lose 20 pounds in a month.'

Developing concrete plans was seen as another way for the PCP and participant to work together. 


\section{Theme 4: Limitations of the Primary Care Setting}

Participants expressed acute awareness of the limitations of their interactions with their PCP, especially related to clinic visits. These included the scope of the visit and the capability of the PCP to provide certain services.

Participants expressed some confusion about the scope of their clinic visits. For example, they only expected to talk about weight management with their PCP during a routine appointment (e.g., a yearly physical) or when they were already in the office for another reason: "You're not going to the doctor because you're overweight or have weight problems. You're going to the doctor because you've got a cold or something else is going on." Participants were hesitant to make an appointment solely for weight management issues because they didn't know if their insurance would cover it: "As I understand it, if you want it covered under your insurance, you better start talking in your [original] 15 min." Participants who could not afford to pay for an appointment out of pocket were even more cautious about making these appointments.

Finally, many participants thought some weight management issues were outside the scope of their PCP. They wanted referrals to nutritionists or dieticians, behavioral therapists, and/or trainers because they felt these professionals were better suited to talk to them about weight management: "I think it takes a team to lose weight. You have to have a dietician, your doctor, and then a psychiatrist. I think it takes a whole group." This was especially true for behavioral issues. Although participants wanted their PCP involved, they recognized potential limits to their PCP's support:

If I go in with questions or concerns about my weight or weight management, I would expect that they would provide me with either referrals or solutions that would actually help me achieve that goal just like they would if I were trying to manage diabetes or high blood pressure or anything else in my life.

Participants felt they had to advocate for themselves to overcome these barriers. One participant who had undergone bariatric surgery said she broached the topic with her PCP:

I went in with a plan, saying, 'This is what I'm interested in doing. Is this an option? Do you think this will work?' ... I felt like I needed to go in very informed to come out with any answers.

Another said, "you gotta ask for the time." Finally, another participant said that when she goes into her annual physical she "bring(s) a list in... and talk(s) very fast" to ensure all her concerns are addressed. This advocacy helped participants partner with their PCP to improve their care. 


\section{Discussion}

This study brings a qualitative patient perspective to the body of literature on weight management in the clinical setting. To date, few studies have examined the patient perspective within in this setting. These findings are unique in complementing research from the provider perspective (Bennett, Gudzune, Appel, \& Clark, 2014; Hebl \& Xu, 2001; Khandalavala, Rojanala, Geske, Koran-Scholl, \& Guck, 2014) as well as past quantitative studies on patient preferences (Amy, Aalborg, Lyons, \& Keranen, 2006; Davis et al., 2006) to expand the picture of what is needed in the clinical setting.

Regarding participants' weight management experiences in the primary care setting, in congruence with the literature, we found that participants were cognizant of the reality of providers' limited time during appointments, and that this constraint prohibited and potentially intimidated patients, thus acting as a barrier to discussing their weight (Davis et al., 2006). Other participants felt they had to lead the discussion and advocate for themselves with their PCP or the issue would not be addressed.

Participants want continuous support and counseling beyond the availability of a PCP appointment. This research provides evidence that participants would be willing to have their PCP function in a supporting role in their weight management. Tailored goal-setting and positive recognition from providers about small changes in a patient's behavior are essential to weight management (Bennett, Gudzune, Appel, \& Clark, 2014). However, participants also believe behavioral providers may be more effective for the kind of goal-setting, consistent accountability, and followup on referrals needed. Participants understood that the ability of their PCPs to provide weight management support might be limited.

The findings of this study are particularly timely because of the 2010 enactment of the Patient Protection and Affordable Care Act. More people than ever are not only covered by insurance but have coverage that includes some weight management counseling and services (American Academy of Family Physicians, 2014). Furthermore, obesity is now classified as a disease (Jensen et al., 2014), which encourages providers to more actively treat patients for obesity and may encourage patients to expect and seek medically supervised care for weight management. Understanding the patient perspective on weight management can guide creation of programs that resonate with patients, thus making behavior change more achievable and potentially improving health outcomes.

For instance, programs that resonate with patients should lead to increased patient engagement. Patient engagement promotes improved health outcomes in the primary care setting (Greene, Hibbard, Sacks, Overton, \& Parrotta, 2015) and with patients who have chronic diseases (Hibbard, Greene, Shi, Mittler, \& Scanlon, 2015; Simmons, Wolever, Bechard, \& Snyderman, 2014). Improvements in patient activation are also associated with positive changes in health behaviors (Hibbard, Mahoney, Stock, \& Tusler, 2007). This study shows patients want to be engaged in weight management. Creating a program that engages patients may help patients 
improve their health outcomes above what would be expected from a typical weight management program in a primary care setting.

Patient engagement is a hallmark of the chronic care model, which addresses the increasing need to broaden the focus of health care to incorporate disease management and prevention (Coleman, Austin, Brach, \& Wagner, 2009; Wagner, Austin, \& Von Korff, 1996). Chronic disease care requires ongoing treatment and support not feasible in a PCP acute visit framework. Wagner's chronic care model suggests a paradigm shift in the patient-provider relationship, placing greater responsibility on patients for decision making and self-management, while providers act as consultants. In this model, education can be provided by the PCP or by a nurse or care manager to enable patients to make informed decisions about their care and to implement strategies that support long term health. The PCP or a care manager coordinates care with specialty providers (e.g., nutritionists, bariatric surgeons, psychologists, cardiologists, pharmacists) when needed, and the care manager might also regularly contact patients to offer support and encouragement for incorporating new daily routines or behavior changes. Real world studies applying this model, including one that used care managers for patients with heart failure and diabetes, have demonstrated both feasibility and positive patient health and self-management outcomes (Ciccone et al., 2010).

The Patient Centered Medical Home (PCMH) is another framework for providing chronic disease care. The medical home provides a number of integrated services, including medical, psychiatric, and social services. Pilot studies show that when behavioral health and medical providers work in integrated care environments, patients experience better access to care, show improved outcomes, and receive more cost effective care than they do from usual care (Amiel \& Pincus, 2011). While behavioral health providers (e.g., psychologists, psychiatrists, social workers, substance use specialists) may offer therapy for mental health disorders, they also specialize in counseling for behavior change, including behavioral change for addictions to substances and obesity. Using this team-based model frees PCPs to address acute medical issues and support longer-term chronic care in collaboration with behavioral health providers. Thus, the chronic care and PCMH integrated health models show potential for addressing the barriers identified by our participants in the acute care model PCP visit.

We note several limitations to this qualitative study. The interviewer effect (e.g., interactions, appearance) could potentially have biased what participants felt comfortable sharing. However, participants seemed grateful and even eager to share their thoughts. While the interviewers made it clear that they did not work for the clinic and every precaution was taken to maintain confidentiality, it is possible that participants had concerns that their responses could negatively affect delivery of care. In terms of sampling, due to the mode of collection, the participants were limited to a geographical radius around the focus group location, and although the sampling frame was representative of patients with obesity at the clinical practice, actual participants were chosen based on a purposive sample. Given this type of sampling, participants who were part of the study may have had different experiences or different motivations for contributing and the external validity of our findings may be limited. That said, the goal of this study was not to seek a 
representative sample but rather to capture experiences in depth. Because there were fewer men in the group, they may have been less vocal than had there been a maleonly group. Finally, all of our participants had a primary care appointment in the previous 12 months, but some participants may have been more engaged with the primary care practice and with weight management than others.

The findings of this focus group study have important implications for supporting patients in managing their weight in order to live healthy and fulfilling lives. Although previous studies have evaluated patient-provider weight management communication practices and physician bias (Caldwell, Gray, \& Wolever, 2013; Campos, 2014; Foster et al., 2003; Phelan et al., 2014; Puhl \& Heuer, 2010; Sherson, Yakes Jimenez, \& Katalanos, 2014), this research enhances those findings by specifying barriers patients face during the primary care visit and components they feel should be considered to create a more effective, patient-centric integrated care model of health delivery. Our findings increase our understanding of different potential program components that patients believe would help them achieve success in weight management. Specific suggestions include realistic outlined plans, means of accountability (e.g., partner, group), communication between PCPs and any in-house or outside weight management assistance, and follow-up. This understanding lays the foundation needed to develop, design and implement a patient-centered, evidence-based pilot weight management program. This program, if successful, could be a model for other primary care practices to ultimately decrease obesity prevalence.

Funding This work was supported by a grant from the Small Grants Program for Research and Scholarship in the Department of Family Medicine at the University of North Carolina at Chapel Hill.

\section{Compliance With Ethical Standards}

Conflict of Interest None of the authors have any conflicts of interest to report.

\section{References}

American Academy of Family Physicians. (2014). AAFP details ACA's 2014 preventive services; Offers Clinical, Coding Resources. Leawood, KS: American Academy of Family Physicians.

Amiel, J. M., \& Pincus, H. A. (2011). The medical home model. Current Opinion in Psychiatry, 24(6), 522-528. https://doi.org/10.1097/YCO.0b013e32834baa97.

Amy, N. K., Aalborg, A., Lyons, P., \& Keranen, L. (2006). Barriers to routine gynecological cancer screening for White and African-American obese women. International Journal of Obesity, 30(1), 147-155. https://doi.org/10.1038/sj.ijo.0803105.

Bennett, W. L., Gudzune, K. A., Appel, L. J., \& Clark, J. M. (2014). Insights from the POWER practicebased weight loss trial: A focus group study on the PCP's role in weight management. Journal of General Internal Medicine, 29(1), 50-58. https://doi.org/10.1007/s11606-013-2562-6.

Caldwell, K. L., Gray, J., \& Wolever, R. Q. (2013). The process of patient empowerment in integrative health coaching: How does it happen? Global Advances in Health and Medicine: Improving Healthcare Outcomes Worldwide, 2(3), 48-57. https://doi.org/10.7453/gahmj.2013.026.

Campos, C. (2014). Tips for communicating with overweight and obese patients. The Journal of Family Practice, 63(7), S11-S14.

Centers for Disease Control and Prevention. (2010). Obesity rises among adults. https://www.cdc.gov/ vitalsigns/pdf/2010-08-vitalsigns.pdf. Accessed 12 July 2018. 
Centers for Disease Control and Prevention. (2014). Obesity prevalence maps. https://www.cdc.gov/ obesity/data/databases.html. Accessed 12 July 2018.

Ciccone, M. M., Aquilino, A., Cortese, F., Scicchitano, P., Sassara, M., Mola, E., et al. (2010). Feasibility and effectiveness of a disease and care management model in the primary health care system for patients with heart failure and diabetes (Project Leonardo). Vascular Health and Risk Management, 6, 297-305.

Coleman, K., Austin, B. T., Brach, C., \& Wagner, E. H. (2009). Evidence on the chronic care model in the new millennium. Health Affairs, 28(1), 75-85. https://doi.org/10.1377/hlthaff.28.1.75.

Davis, N. J., Emerenini, A., \& Wylie-Rosett, J. (2006). Obesity management: Physician practice patterns and patient preference. The Diabetes Educator, 32(4), 557-561. https://doi.org/10.1177/ 0145721706290437.

Foster, G. D., Wadden, T. A., Makris, A. P., Davidson, D., Sanderson, R. S., Allison, D. B., et al. (2003). Primary care physicians' attitudes about obesity and its treatment. Obesity Research, 11(10), 1168-1177.

Greene, J., Hibbard, J. H., Sacks, R., Overton, V., \& Parrotta, C. D. (2015). When patient activation levels change, health outcomes and costs change, too. Health Affairs, 34, 431-437. https://doi.org/10.1377/ hlthaff.2014.0452.

Greiner, K. A., Born, W., Hall, S., Hou, Q., Kimminau, K. S., \& Ahluwalia, J. S. (2008). Discussing weight with obese primary care patients: Physician and patient perceptions. Journal of General Internal Medicine, 23(5), 581-587. https://doi.org/10.1007/s11606-008-0553-9.

Gudzune, K. A., Bennett, W. L., Cooper, L. A., \& Bleich, S. N. (2014). Perceived judgment about weight can negatively influence weight loss: A cross-sectional study of overweight and obese patients. Preventive Medicine, 62, 103-107.

Hebl, M. R., \& Xu, J. (2001). Weighing the care: Physicians' reactions to the size of a patient. International Journal of Obesity and Related Metabolic Disorders, 25(8), 1246-1252. https://doi. org/10.1038/sj.ijo.0801681.

Hibbard, J. H., Greene, J., Shi, Y., Mittler, J., \& Scanlon, D. (2015). Taking the long view: How well do patient activation scores predict outcomes four years later? Medical Care Research and Review. https://doi.org/10.1177/1077558715573871.

Hibbard, J. H., Mahoney, E. R., Stock, R., \& Tusler, M. (2007). Do increases in patient activation result in improved self-management behaviors? Health Services Research, 42, 1443-1463. https://doi.org/10. 1111/j.1475-6773.2006.00669.x.

Jensen, M. D., Ryan, D. H., Apovian, C. M., Ard, J. D., Comuzzie, A. G., Donato, K. A., et al. (2014). 2013 AHA/ACC/TOS guideline for the management of overweight and obesity in adults: A report of the American College of Cardiology/American Heart Association task force on practice guidelines and the obesity society. Journal of the American College of Cardiology, 63(25 Pt B), 2985-3023. https://doi.org/10.1016/j.jacc.2013.11.004.

Khandalavala, B. N., Rojanala, A., Geske, J. A., Koran-Scholl, J. B., \& Guck, T. P. (2014). Obesity bias in primary care providers. Family Medicine, 46(7), 532-535.

LeBlanc, E., O'Connor, E., Whitlock, E., Patnode, C., \& Kapka, T. (2011a). Effectiveness of primary care-relevant treatments for obesity in adults: A systematic evidence review for the U.S. Preventive Services Task Force. Annals of Internal Medicine, 155, 434-437.

LeBlanc, E., O'Connor, E., Whitlock, E., Patnode, C., \& Kapka, T. (2011b). Screening for and management of obesity and overweight in adults. Evidence synthesis. Rockville, MD: Agency for Healthcare Research and Quality.

Miles, M., \& Huberman, A. (1994). Qualitative data analysis: An expanded source book (2nd ed.). London: Sage.

Phelan, S. M., Dovidio, J. F., Puhl, R. M., Burgess, D. J., Nelson, D. B., Yeazel, M. W., et al. (2014). Implicit and explicit weight bias in a national sample of 4,732 medical students: The medical student CHANGES study. Obesity (Silver Spring, Md.), 22(4), 1201-1208. https://doi.org/10.1002/oby. 20687.

Puhl, R. M., \& Heuer, C. A. (2010). Obesity stigma: Important considerations for public health. American Journal of Public Health, 100(6), 1019-1028. https://doi.org/10.2105/AJPH.2009.159491.

Rice, P., \& Ezzy, D. (1999). Qualitative research methods: A health focus (1st ed.). Oxford: Oxford University Press.

Sherson, E. A., Yakes Jimenez, E., \& Katalanos, N. (2014). A review of the use of the 5 A's model for weight loss counselling: Differences between physician practice and patient demand. Family Practice, 31(4), 389-398. https://doi.org/10.1093/fampra/cmu020. 
Simmons, L. A., Wolever, R. Q., Bechard, E. M., \& Snyderman, R. (2014). Patient engagement as a risk factor in personalized health care: A systematic review of the literature on chronic disease. Genome Medicine, 6(2), 16. https://doi.org/10.1186/gm533.

US Preventive Services Task Force. (2012). Final recommendation statement: obesity in adults: screening and management. Retrieved from http://www.uspreventiveservicestaskforce.org/Page/Document/ RecommendationStatementFinal/obesity-in-adults-screening-and-management. Accessed 12 July 2018.

Vallis, M., Piccinini-Vallis, H., Sharma, A., \& Freedhoff, Y. (2013). Modified 5 as minimal intervention for obesity counseling in primary care. Canadian Family Physician, 59(1), 27-31.

Wadden, T. A., Anderson, D. A., Foster, G. D., Bennett, A., Steinberg, C., \& Sarwer, D. B. (2000). Obese women's perceptions of their physicians' weight management attitudes and practices. Archives of Family Medicine, 9(9), 854-860.

Wagner, E. H., Austin, B. T., \& Von Korff, M. (1996). Organizing care for patients with chronic illness. The Milbank Quarterly, 74(4), 511-544.

\section{Affiliations}

\section{Kate Bloom ${ }^{1} \cdot$ Jaime Adler $^{1} \cdot$ Christy Bridges $^{1} \cdot$ Julia Bernstein $^{1} \cdot$} Christine Rini ${ }^{2} \cdot$ Adam O. Goldstein $^{3,4} \cdot$ Carol Ripley-Moffitt ${ }^{3}$

Carol Ripley-Moffitt

carol_ripley-moffitt@med.unc.edu

1 Department of Health Behavior, Gillings School of Global Public Health, University of North Carolina at Chapel Hill, Chapel Hill, NC, USA

2 John Theurer Cancer Center, Hackensack University Medical Center, Hackensack, NJ, USA

3 Department of Family Medicine, University of North Carolina at Chapel Hill, 590 Manning Drive, CB \#7595, Chapel Hill, NC 27599-7595, USA

4 UNC Lineberger Comprehensive Cancer Center, School of Medicine, University of North Carolina at Chapel Hill, Chapel Hill, NC, USA 